\title{
Direct Complication of HIV on the Brain: A Case About a Recurrence of Stroke
}

\section{Abstract}

Only $1 \%$ to $5 \%$ of HIV patients who develop a direct complications following. HIV infection can result in stroke via several mechanisms, including opportunistic infection, vasculopathy, cardioembolism and coagulopathy. It is a rare association, here we report the case of a woman of 69 years immunocompromised who Stroke diagnosed without another etiology found despite an etiologic assessment of the most common causes in our context. The patient had received treatment with an overall favorable evolution. Before a stroke recurrent of unknown etiology should think of HIV as this will allow appropriate treatment. The mechanism can be related with syphilis (Vasculitis) or by direct action of the virus on the central nervous system.

Keywords: HIV; Stroke; Recurrence; Senegal

Received: January 12, 2017; Accepted: March 10, 2017; Published: March 17, 2017

\section{Introduction}

Between $1 \%$ and $5 \%$ of patients with HIV develop stroke in clinical series, although a higher proportion (4-34\%) have cerebral lesions at autopsy public health [1]. HIV infection can result in stroke via several mechanisms, including opportunistic infection, vasculopathy, cardioembolism and coagulopathy [2]. This association of HIV and STROKE is rare, we report here a case of recurrence of STROKE associated with HIV.

\section{Case Report}

It was a 69 year old, right-handed woman, with a history of HIV 2 since 2001, high blood pressure and ischemic STROKE. She was received in our Neurology department in October 2016 for motor deficit of the left hemibody with language of appearance brutal disorder. Clinical examination has objectified a score of Glasgow to $11 / 15$, left hemiplegia, a rough right hemiparesis. The rest of the clinical examination was without notable features. The brain scan revealed the presence of 2 homes of hypodensity in the level of the territories of the right and left sylviennes arteries associated with cortico-under cortical atrophy and a Leucoareose (Figure 1).

Transthoracic ultrasonography of even transoesophagienne was within normal limits. The electrocardiogram, holter 24 hours, and the brain angioscanner have been normal. The vessels of the neck ultrasound showed a diffuse bilateral carotid intimo-medial thickening without hemodynamic effect. The

\author{
Ousmane Cisse', \\ Soumaila Boubacar ${ }^{1}$, \\ Ibrahima M Diallo', \\ Samy LM Dadah', \\ Patrice Ntenga ${ }^{1}$, \\ Kalidou Diallo², \\ Marieme S Diop-Sène ${ }^{1}$, \\ El Hadji M Ba', \\ Adjaratou D Sow', \\ Anna M Basse', \\ Noel M Manga², \\ Moustapha Ndiaye', \\ Amadou G Diop' and \\ Mouhamadou M Ndiaye ${ }^{1}$
}

1 Department of Neurology, Fann National Teaching Hospital, Dakar, Senegal

2 Hospital De La Paix of Ziguinchor, Senegal

Corresponding author: Ousmane Cisse

” ouscis01@hotmail.fr

Department of Neurology, Fann National Teaching Hospital, Dakar, Senegal.

Tel: + 221775078751

Citation: Cisse O, Boubacar S, Diallo IM, et al. Direct Complication of HIV on the Brain: A Case About a Recurrence of Stroke. J HIV Retrovirus. 2017, 3:1.

lumbar puncture brought back a clear liquid, with 23 items (to lymphocytic predominance) and $0.93 \mathrm{~g} / \mathrm{l}$ of content as the FTA (Fluorescent Treponemal Antibody) was negative. Serology positive HIV2, the CD4 count at 263 viral load: 48.000 copies/ $\mathrm{ml}$ Antigen $\mathrm{Hbc}$ (negative), syphilitic serology and Antigen Hbs (negative). The rest of the balance of risk and Etiologic factors was normal. Before these clinical disorders of the patient and the check carried out, we had retained the diagnosis of recurrence of stroke on high blood pressure and field of immunosuppression (HIV 2). The patient had a treatment with acetylsalicylic acid, antihypertensive (inhibitor of the enzyme conversion, combined 


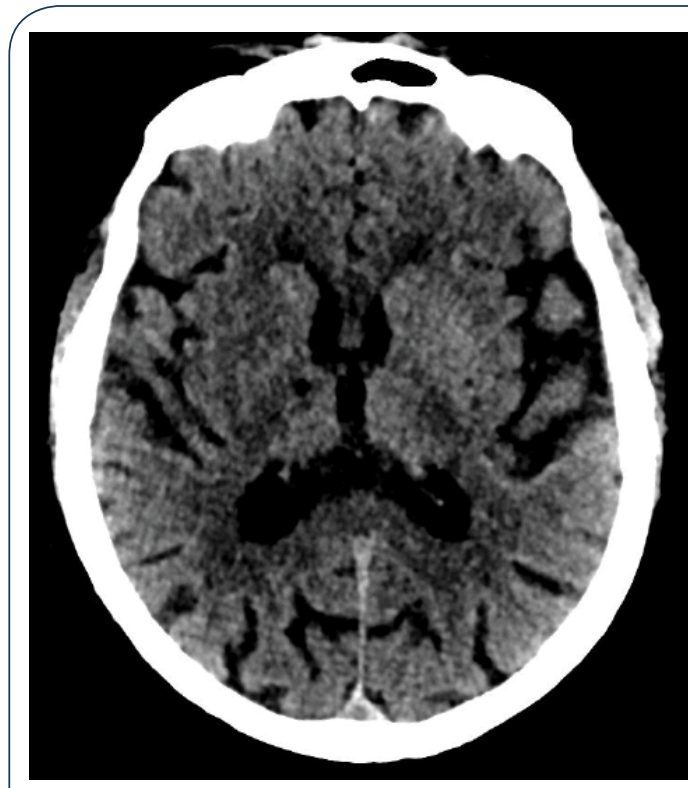

Figure 1 Brain scan revealed the presence of 2 homes of hypodensity in the level of the territories of the right and left sylviennes arteries associated with cortico-under cortical atrophy and a leucoareose.

with a calcium inhibitor), enoxaparin sodium 4000 IU anti-Xa per day, physiotherapy, Ceftriaxone 2 g per day, strict monitoring of vital parameters. She had benefited from the association zidovudine lamivudine (Combivir ${ }^{\circledast}$ ) in the dose of one tablet twice a day, but she was more back until her episode of stroke. After counseling on compliance the same antiretroviral treatment has been extended. The patient was still not regularly his treatment.

\section{Discussion}

The stroke in general presents a rather singular symptomatology with a brutality of installation of signs. If brain imaging (scanner or magnetic resonance imaging allows to differentiate between the haemorrhage and ischaemia, the search for the cause requires more resources, especially if ischemia [3]. The most frequent etiologies are heart disease emboligenes and atheromatous infiltration [3]. Otherwise, exceptional and rare causes are possible [3]. We report a case of ischemic stroke recurrent related with infection by HIV 2. The diagnosis of stroke related to HIV was retained after eliminating the most common cause's context and some rare causes According to the classification of TOAST.

HIV has dominated neurological complications by opportunistic infections including neurologically [4]. However with the advent of antiretroviral treatment, but also the use of antibiotics seems to decrease these types of complication and may be causing others 'direct' said complications from the virus. The stroke associated with HIV seems to be a rare and exceptional complication.

These Strokes are multifactorial due to a State of hypercoagulability its HIV him even through including a drop of protein $\mathrm{C}$, an inflammatory condition extended from opportunistic infections and by iatrogenic effect of anti proteases [5]. This may be at the origin of venous ischemia [6] or pressure like that seems to be the case for our patient. The other hypothesis referred to the intervention of a strong emergence of syphilis because of the advent of HIV [7]. That was not the case in our patient. Indeed the syphilitic serology was negative in blood and cerebrospinal fluid. The presence of recurrence shows the HIV Embolic potential and conditions effective treatment. Our patient had continued its HIV antiretrovial treatment. The evolution was generally favorable.

\section{Conclusion}

Before a stroke recurrent of unknown etiology should think of HIV as this will allow appropriate treatment. Indeed, there are various causes of stroke and HIV seems to be potentially embolic recurrence where noted in our observation. The mechanism can be related with syphilis (Vasculitis) or by direct action of the virus on the central nervous system.

\section{Conflict of Interest}

No 


\section{References}

1 Kieburtz KD, Eskin TA, Ketonen L, Tuite MJ (1993) Opportunistic cerebral vasculopathy and stroke in patients with the acquired immunodeficiency syndrome. Arch Neurol 50: 430-432.

2 Benjamin LA, Bryer A, Emsley HCA, Khoo S, Solomon T, et al. (2012) HIV infection and stroke: Current perspectives and future directions. Lancet Neurol 11: 878-890.

3 Uchino K, Pary J, Grotta J (2009) Accident vasculaire cérébral ischémique. Urgences neurovasculaires, pp: 15-38.

4 Lacroix C (1999) Manifestations neurologiques du VIH. EMC. Paris: Elsevier, Neurologie, p: 11.
5 Tazi-Mezalek ZT, Alaoui-Bennasser H, Maamar M, Harmouche $\mathrm{H}$, Adnaoui M (2014) Thrombose et infection a' VIH : A propos de 10 cas. J Mal Vasc 39: 351-352.

6 Dadah SML, Gaye NM, Diop A, Diagne NS, Diop MS, et al. (2015) Thromboses veineuses cérébrales multiples révélatrices d'une infection à VIH. Rev Neurol 171: 736-737.

7 Lachaud S, Suissa L, Mahagne MH (2010) Infarctus cérébral, infection VIH et syphilis méni ngovasculaire: étude de trois cas. revue neurologique 166 : $76-82$. 